\title{
Functional foods and dietary supplements
}

\author{
Melissa M. Phillips • Catherine A. Rimmer
}

Published online: 14 March 2013

(C) Springer-Verlag Berlin Heidelberg (outside the USA) 2013

General interest in improving or maintaining health by increasing intake of "all-natural" and "organic" products combined with busier schedules has created a desire for more streamlined approaches to nutrition. Analysis of data from the 2007-2010 National Health and Nutrition Examination Survey (NHANES) revealed that $49 \%$ of US adults reported using a supplement within 30 days of the study [1]. The main reasons for taking supplements were identified as being to "improve overall health" (45\%) and to "maintain health" (33\%) [1]. The result has been growth in the areas of functional foods and dietary supplements, both in product variety and sales.

Functional foods and dietary supplements are two of the major classes of nutraceuticals or food-related products that have purported health or medical benefits, such as improving cardiovascular health and prevention or treatment of disease. Functional foods can include foods that have been either enriched or fortified to restore preprocessed nutrient levels (enriched flour), to improve nutritional quality of an otherwise nutrient-deficient food (calcium in orange juice), or to resolve

Published in the topical collection Functional Foods and Dietary Supplements with guest editors Melissa M. Phillips and Catherine A. Rimmer.

Certain commercial equipment, instruments, or materials are identified in this paper to specify adequately the experimental procedure. Such identification does not imply recommendation or endorsement by the National Institute of Standards and Technology, nor does it imply that the materials or equipment identified are necessarily the best available for the purpose.

M. M. Phillips $(\bowtie) \cdot$ C. A. Rimmer

National Institute of Standards and Technology, 100 Bureau Drive,

Mailstop 8392,

Gaithersburg, MD 20899-8392, USA

e-mail: melissa.phillips@nist.gov

C. A. Rimmer

e-mail: catherine.rimmer@nist.gov public health issues (vitamin D in milk, iodized table salt). Some consider a functional food to be any food that provides health benefits beyond basic nutrition, such as the soluble fiber found in oatmeal and the monounsaturated fat found in avocados. Studies are ongoing to identify functional characteristics of traditional foods and to develop new products that exploit beneficial components. A recent survey indicates that two thirds of US grocery shoppers have purchased a food or beverage specifically to address one of a number of common health concerns (e.g., cholesterol, digestive health) [2].

Dietary supplements, including concentrated forms of food-derived nutrients, are the other major class of nutraceuticals. Unlike functional foods, dietary supplements are not intended to be food or meal replacements, but are designed to be taken in addition to daily food consumption for added nutrients or perceived health benefits. Global dietary supplement sales continue to rise, growing $6.1 \%$ in 2011 , from $\$ 79.43$ billion to $\$ 84.26$ billion [3]. The most common dietary supplements are multivitamin-mineral products, calcium, $\omega-3 /$ fish oil, and botanicals [1]. Botanicals are a growing category, however, as the botanical and natural ingredient export trade reached $\$ 33$ billion in 2010, with projections of up to $\$ 100$ billion by 2015 [4].

With a large market share and variety of products comes a need for quality control and regulation. For functional foods, the approach to marketing determines the regulatory category. In the USA, some functional foods are regulated as foods, falling under the Nutritional Labeling and Education Act of 1990 [5]. In other cases, functional foods are regulated as dietary supplements. The current Good Manufacturing Practices (cGMPs), published by the US Food and Drug Administration in 2010, require that dietary supplements produced in the USA are evaluated for identity, purity, and strength as well as for contaminants and adulterants [6]. Quality measurements are also important when establishing efficacy as a clinical trial is much more persuasive when active ingredients and their 
metabolites are quantified both pre- and post-consumption. To both ends, validated analytical methods and quality control materials are important tools, the development of which has been advocated by the Analytical Methods and Reference Materials Program in the Office of Dietary Supplements at the US National Institutes of Health [7].

The need for analytical methods and clinical trials for functional foods and dietary supplements has been noted by the scientific community. In a search in Web of Knowledge (Thomson Reuters) for publications in the last 5 years (2009-2013) the terms "functional food" or "dietary supplement" returned over 15,000 publications. This number has grown exponentially since the early 1990 s, and the trend predicts over 4,700 publications to come in 2013. This special collection of papers in Analytical and Bioanalytical Chemistry includes contributions from industry, academia, and government related to the analytical challenges associated with the biological, analytical, and regulatory aspects of nutraceutical preparation and analysis. Much of the current research focuses on high-resolution mass spectrometry approaches to material characterization and authentication as well as various aspects of quality control. This collection is an excellent representation of the current research effort in the area of functional foods and dietary supplements, and we hope you enjoy its contents. These research areas have made great strides in recent years, but the constantly changing marketplace leaves many important questions unanswered.

\section{References}

1. Bailey RL, Gahche JJ, Miller PE, Thomas PR, Dwyer JT (2013) JAMA Intern Med doi:10.1001/jamainternmed.2013.2299
2. Sprinkle D (2012) Nutraceuticals World 15:32-37

3. Zambetti P (2012) Nutraceuticals World 15:37-42

4. Dennis J (2012) Nutraceuticals World 15:46-53

5. US FDA (1990) Nutrition labeling and education act. Public Law 101-535 [H.R. 3562] Nov 81990.

6. FDA (2007) Current good manufacturing practice in manufacturing, packaging, labeling, or holding operations for dietary supplements. Docket no. 1996N-0417 (formerly no. 96N-0417), 34752-34958. 625-21 CFR Part 111.

7. Betz JM, Fisher KD, Saldanha LG, Coates PM (2007) Anal Bioanal Chem 389:19-25

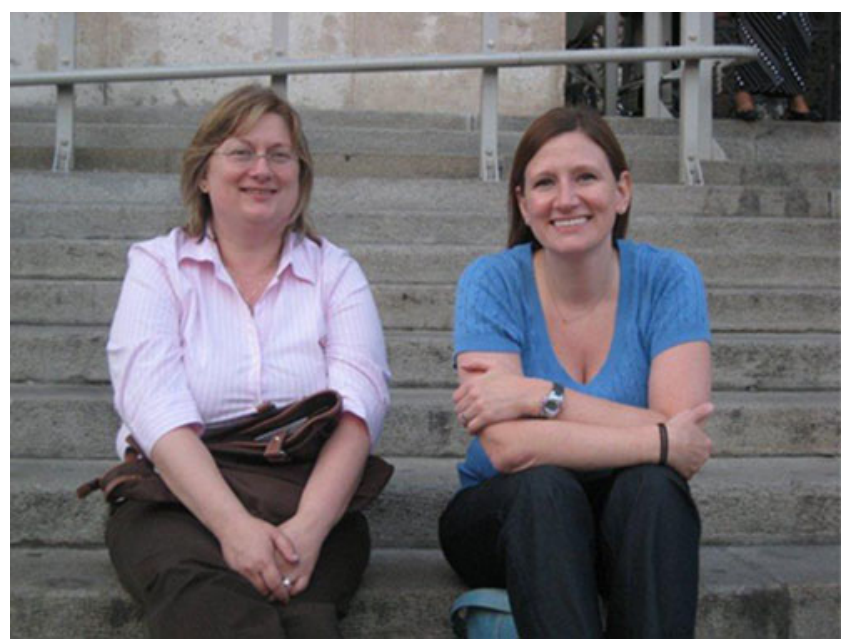

Catherine A. Rimmer (left) and Melissa M. Phillips are research chemists at the National Institute of Standards and Technology in Gaithersburg, MD, USA. They are involved in the certification efforts for food and dietary supplement Standard Reference Materials and are co-coordinators of the Dietary Supplement Laboratory Quality Assurance Program. Their interests include development of new analytical methods for the determination of marker compounds and vitamins in dietary supplements and foods, as well as improving the measurement capabilities of the dietary supplement and food communities. Here, they are enjoying the views from the steps of St. Stephen's Basilica in Budapest, Hungary. 